\title{
UK Construction Safety: A Zero Paradox?
}

Fred Sherratt ${ }^{1}$ and Andrew R. J. Dainty ${ }^{2}$

${ }^{1}$ Department of Engineering and the Built Environment, Anglia Ruskin University, Chelmsford, UK

${ }^{2}$ School of Civil and Building Engineering, Loughborough University, Loughborough, $U K$

Corresponding Author: Dr Fred Sherratt, Anglia Ruskin University, Bishop Hall Lane, Chelmsford, CM1 1SQ, UK. 01245 683950, fred.sherratt@anglia.ac.uk 


\section{UK Construction Safety: A Zero Paradox?}

The zero accident mantra has become embedded within the safety discourse of large UK construction organisations, but the extent to which zero-focused approaches yield reductions in accident frequency is yet to be empirically investigated. By way of an evidence-based critique, we examine the relationship between major accidents and zero approaches by drawing on Health and Safety Executive accident data over a 4 year period, together with an analysis of major contractors' safety approaches. This reveals that working on a project subject to a zero safety policy or programme actually appears to slightly increase the likelihood of having a serious life-changing accident or fatality; a possible 'zero paradox'. Although these findings should be treated with caution, they suggest that the apparent trend towards abandoning zero amongst some large organisations is well-founded. As such, if zero policies stymie learning whilst failing to reduce accidents, the need for a countervailing discourse is clear.

Keywords: construction industry, safety, United Kingdom, zero paradox, zero

\section{Introduction}

Zero has become the biggest number in safety (Sherratt, 2014). It is becoming more prominent in academia, where it has found both critics, uncomfortable with the theoretical, philosophical and discursive consequences of its emergence (c.f. Dekker, 2014; Dekker, Long and Wybo, 2016; Hollnagel, 2015; Long, 2012; Sherratt, 2014), and champions, who stress the differences between targets and visions (c.f. Zwetsloot, Aaltonen, Wybo, Saari and Kines, 2013, Zwetsloot, Kines, Wybo, Ruotsala and Drupsteen, 2017) and align it to other, more practical and tangible, developments in safety management with clear beneficial consequences (c.f. Young, 2012). Zero has permeated the safety management systems of many different organisations within petrochemical, manufacturing and engineering industries. Indeed, it has seemingly become an inherent part of the way safety is managed and delivered in practice; as Long (2012, p.11) points out, such organisations no longer employ Safety Managers but 
applications are now sought for 'Zero Harm Advisors', potentially a consequence of the growing influence of marketing and public relations in safety management (Sherratt, 2016a).

Yet it is perhaps not the labelling of Zero, or its philosophical implications or semantic nuances that really matter. It could even be argued that what academics and corporate leaders think Zero means does not really matter very much either. What actually matters is the extent to which Zero tangibly influences managerial, administrative or operative safety practices that, in turn, affect safety outcomes. Indeed, the lack of an evidenced-based evaluation of Zero is surprising given the amount of interest in its emergence in recent years as Zwestsloot et al (2017. p.264) argue in their defence of the Zero Harm Vision that there has been a lack of ' ...empirical research published in peer reviewed journals' from those who critique Zero, that there is no '... strong scientific case' that Zero has unintended consequences or negative effects on practice. This paper seeks to redress this by presenting a case study of a single hazardous sector; the UK construction industry. Specifically, we critically examine the relationship between the mobilisation of Zero within organisational safety management and the actual numbers of potentially life-changing incidents that occurred on those organisations' construction sites over a four year period. Whilst no claim is made to causality, analysis of this data reveals a potential Zero paradox: statistically, you are marginally more likely to have major/specified accident working on a large construction site operated by a company that has adopted Zero within their health and safety strategy, than if you are working on a site without it.

\section{The UK Construction Industry and Zero}

It is often suggested that construction is not like any other industry in terms of health and safety management, in part because of the inherent hazards and risks 
associated with construction work itself, yet consideration should also be given to the wider way construction work is structured, and the pressures this also brings to safety management on sites.

\section{A Hazardous Workplace}

On construction sites, the work environment is constantly changing and growing as work progresses. This in turn inevitably creates the need for workers to be in close proximity to excavations, leading edges and heights, as foundations, walls and roofs are constructed. This creates a wide variety of different workplace hazards, which are also in constant development as the next work within the project begins. In order to carry out such work, heavy and potentially dangerous plant, machinery and equipment are in constant use, whilst toxic materials and chemicals are also frequently used.

Construction sites present an ever-changing work space, where hazards are an inevitable part of the process, and their careful and continuous management within the site context is therefore an essential task (Sherratt, 2016b).

\section{Building in Unsafety}

In addition to the hazards found on the site itself, it has also been argued that the very way the industry operates is the fundamental cause of its poor safety record (Cipolla, Sheahan, Biggs and Dingsdag, 2006). The way work is awarded to construction companies through competitive tendering processes can mean a focus on the direct cost of the labour, plant and materials for construction rather than other project considerations, such as safety (Lingard and Rowlinson, 2005). Due in part to the project-based nature of the work, there is also a reliance on subcontracting, which often sees long industry supply chains working on large sites, which also has negative consequences for safety (Manu, Ankrah, Proverbs and Suresh, 2010). The same 
tendering processes are also used to establish industry supply chains, and so the pressures on profit are squeezed tighter and tighter along them, reducing the money available for safety management and training (Lingard and Rowlinson 2005) within supply chain organisations, and it is often the smaller contractors who are most vulnerable to poor safety practices (Donaghy, 2009), as they strive to win work within such constrictive financial environments.

Such a fragmented industry structure has in turn developed a fragmented workforce that is transient and temporary, with little responsibility for or even acceptance of safety management in practice (Seymour and Fellows 2002; Donaghy, 2009). This workforce is also incentivised by bonus and payment schemes that encourage speed and risk taking behaviours (Fellows, Langford, Newcombe and Urry, 2002), paid for the volume of work they produce, rather than whether they produce it safely. The pressures of time and cost can be seen to readily cascade throughout the industry, as it seeks to operate as quickly and cheaply as possible (Sherratt 2016b)to meet the constant demand for production (Health and Safety Executive, 2009; Lingard and Rowlinson, 2005). Indeed, one quarter of the experts consulted for the Donaghy Report (2009) to UK Government, titled 'One Death is Too Many', felt that the way the industry is set up and work is procured has created an ethos that actively encourages safety accidents and incidents.

\section{The Cost of Construction Safety}

The challenges of managing such complexities effectively are sadly reflected in the human cost of construction work; it has been estimated that around 60,000 people die on construction sites worldwide each year (Smallwood and Lingard, 2009). Seen in this context, the UK construction industry is one of the safest in the world. Compared to International Labour Organisation estimates from 2008, where construction worker 
fatality rates ranged from 3.3 to 10.6 deaths per 100,000 workers within certain industrialised countries, the UK rate was only 1.62 per 100,000 workers in 2016 (Health and Safety Executive, 2016) and there is an ongoing quest amongst large construction contractors operating in the UK to improve this even further. To this end, and reflecting wider safety management thinking, many large contractors have enthusiastically adopted Zero into their UK site safety management programmes, embedding its rhetoric in their health and safety policies and strategies.

\section{Construction Safety and Zero}

Zero has been mobilised in a variety of ways within the construction industry, for some 'Beyond Zero' is specifically positioned as a 'visionary journey', adopted as a principle of organisational management and leadership rather than a specific goal, for others 'Zero Harm’ is a specific target, with zero fatalities, zero permanent disabling injuries and zero accidents and injuries. In her research on Zero specifically within this context, Sherratt (2014) found that corporate programmes often position Zero as a tangible goal, a firm 'future perfect' reality (cf. Pitsis, Clegg, Marosszeky, and RuraPolley, 2003), which can be counted and measured through a plethora of targets. Yet this utopia was challenged and even derided by the construction workers themselves, for whom the lived realities of their working lives tells them Zero is, and is likely to remain, a utopian fantasy, totally incompatible with the current challenges of production that they face on a daily basis (Sherratt 2014). Worse still, it might actually stymie the open dialogue and learning culture widely acknowledged as a hallmark of a progressive safety discourse (Long, 2012).

And this is where Zwetsloot et al (2017) would suggest that the UK construction industry has got it wrong; that it has focused on the numbers and not the vision that should inspire real change in practice and catalyse the process of 'innovating to zero' 
(ibid 2017, p.263). Yet such innovative change cannot readily be identified in UK construction safety management; there has been a lack of identifiable development in work practices beyond the simple application of 'Brand Zero'. As within the wider discourse around Zero and safety, there have recently been ripples of a sea-change in industry thinking. Indeed, leading construction industry experts have recently suggested that the application of Zero targets to safety is actually hindering safety management innovation , and has rendered it a bureaucratic activity (Green, 2016), a consideration firmly grounded in the theoretical work of Dekker (2014). Indeed, such a focus on measurement does not seek to challenge and change current practice; rather it aims to operate within the existing environment, seeking engagement of the workforce without addressing problems of practice, creating a distraction, a focus on the numbers and continuous improvement, rather than the practices and the people behind them (Sherratt, 2016b).

Although Zwetsloot et al (2017, p.263) are at pains to point out that 'the exact nature of the beliefs behind ZAV (Zero Accident Vision) are not very interesting, we are interested in the consequences of ZAV: the commitments that generate actions and programmes to develop greater safety, often using or developing innovative means’ (their emphasis), it would seem that for UK construction although such beliefs may not be 'interesting', they hold significant influence to practice. They are precisely what makes Zero in any form a distraction, because of the allure of its measurement (Hollnagel, 2014, p.71), creating a misdirection in efforts to cease all harm rather than the harm that really matters (Dekker et al., 2016, p.222), and stifling both learning and reporting in an industry which already struggles to report its accidents and incidents in an honest and timely fashion (Donaghy, 2009; Long and Long, 2012). It also disengages those to whom it should matter most, the construction site workers. Whilst 
they are happy to position zero as a vision for the future, rather than a target, they remain firmly derisive of its achievement within their current working contexts; for them, a construction site with zero accidents is just a castle in the sky (Sherratt, 2014).

. This paper seeks to subject this particular context of Zero to an evidence-based critique and a degree of empirical scrutiny. It establishes whether large construction organisations operating in the UK that have adopted Zero have seen improvements over those who have not, whether have they developed 'greater safety' in practice, and whether they are able to show any evidence of beginning the process of 'innovating to Zero’.

\section{Method}

Fatal and major/specified accident data for the UK construction industry in the periods 2011-12 to 2014-15 were obtained under a Freedom of Information Request (FOI: 201606138) to the UK Health and Safety Executive (HSE). This data recorded $n=6995$ major/specified and fatal incidents as reported within the UK industry during this time period under UK law. The term 'specified injury' replaces the previous categorisation of 'major injuries' as noted in the Reporting of Injuries, Diseases and Dangerous Occurrences Regulations 2013. Such injuries include amputations, reductions in sight, crush injuries, significant burns and any loss of consciousness (HSE, 2016), all of which have the potential to be life-changing for the injured party. The FOI data also included the name of the reporting company, which in the case of accidents occurring on large construction sites would most likely be the Principal Contractor, as defined by the Construction (Design and Management) Regulations 2015.

There are several important caveats to be considered in the use of this FOI data. The data for the period 2014-15 is still provisional, and so should be considered as such. There may also be 'gaps' in the data, specifically that fatal injuries are not always 
reported via the online system from which this FOI data was generated, but instead '.. by the emergency services or others who see it as their responsibility to do so'. In addition, this dataset does not include incidents reported by the self-employed, due to data protection issues. Due to the nature of subcontracting and the presence of long supply chains on larger projects, there is also the potential that a contractor could be in charge of a site on which an accident occurred, yet not be recorded as the reporting company within this dataset. These caveats can, however, all be recognised as only having the potential to reduce the total number of accidents that actually occurred on large sites under the management of the named Principal Contractors. They are unable to skew the data in the opposite direction and suggest that more accidents of the noted severity occurred on large sites than did in reality. Therefore, despite such issues of validity within the dataset, it can still be used to explore the possible consequences of zero in practice.

The Top 20 contractors working in the UK by turnover were identified through Building Magazine’s ‘Top 150 contractors and housebuilder - Ranked by Turnover’ [20 July 2016]. Data from the period 2014/15 was used, to correlate with the accident data. The turnover of the UK construction industry in 2015 was $£ 135.1 \mathrm{bn}$, and the turnover of these Top 20 contractors combined was £52.5bn (Office of National Statistics, 2015), representing over 39\% of all UK construction work by value. Although these contractors number only 20, subcontracting practices means they will have influence on many sub-contractors and suppliers who work under their safety programmes on the large projects that make up over a third of all UK construction work by value.

A blended approach of content analysis (Ali, 2006) was used to examine company websites and sustainability reports to determine each contractor's approach to zero and safety. This established whether they had an explicit zero safety policy or 
programme, for example ‘Target Zero’ or 'Zero Harm’, or made reference to zerooriented metrics within their health and safety reporting. Historic reports were also examined, to determine the date Zero first appeared within each contractor's approach to health and safety management. The decision of whether a contractor exhibited the traits of a zero accident policy was made independently by both authors in order to corroborate their approach.

Within the accident data a search was carried out for each Top 20 contractor by name, including their operation as part of any joint-venture projects. The numbers of fatal and specified/major injuries each contractor reported in the period 2011-12 to 2014-15 was then correlated with their approach to Zero safety ${ }^{1}$.

\section{Findings}

Analysis of the Top 20 company data found that 9 had an explicit Zero policy in place. 6 of these companies were operating a safety programme referencing zero, whilst the other 3 included clear statements around zero, for example that zero was either a target within their wider programme, or specifically referenced 'incident and injury free'. When this data is correlated with the FOI accident data for the period 2011/12-2014/15, the following is initially revealed:

- $\quad$ There were 4 fatal accidents for companies with zero safety.

- There were 0 fatal accidents for companies without zero safety.

\footnotetext{
${ }^{1}$ It should be noted here that the vast majority of accidents within the dataset did not occur on the sites of these Top 20 contractors. Indeed, in the UK construction health and safety is much more of a concern on small sites and amongst small and micro-organisations. However, it is amongst these Top 20 organisations that Zero has emerged within construction site safety, and so it is this data that has been extracted for analysis.
} 
Furthermore, with regard to major/specified injuries a similar pattern emerges from the data within the period 2011/12-2014/15:

- There were 214 major/specified injuries for companies with zero safety

- There were 135 major/specified injuries for companies without zero safety

Again, and notwithstanding the deficiencies in the dataset outlined above, this does not demonstrate the realisation of the most tangible aspiration of zero in practice. However, these statistics do not reflect the variations in volume of work carried out by these companies, which can be most readily ascertained through their published turnover. The UK turnover of these 9 zero safety companies in 2015 was $£ 29.1 \mathrm{bn}$, representing nearly a quarter (22\%) of all UK construction work. The turnover of the 11 companies operating without zero safety was $£ 23.4 \mathrm{bn}$ (a difference of $£ 5.7 \mathrm{bn}$ ( $4 \%$ of turnover) between the two groups). Although turnover is not the usual metric employed in accident analyses (hours worked is the value used in the production of the more familiar Accident Frequency Rates or AFRs), it is arguably still linked to the total volume of work carried out on sites under the management of these companies. Furthermore, given the UK construction marketplace, it can also be suggested that the types and value of projects undertaken by these companies will to a large extent be comparable within this turnover figure, although it must be noted that here it is used as a proxy

If these statistics are then correlated, the following is revealed:

- $\quad$ There were 7 Fatal or Major/Specified accidents per billion turnover for those with zero safety

- There were 6 Major/Specified accidents per billion turnover for those without zero safety 
Taken together, this analysis suggests the possibility of a 'Zero Paradox' in play within construction site safety for large firms: you are marginally more likely to have major/specified accident whilst working on a large construction site operated by a contractor mobilising any form of Zero safety, than if you are working on a site without it. Zero, for construction on large UK sites, actually means a greater risk of injury (or death) in practice.

In order to provide a consideration of timescales to better frame this data, the content analysis was able to reveal when each company first introduced zero to its wider health and safety practices, for only one organisation (company S) was this data unobtainable. When this data was again correlated against the accident statistics on a year-by-year basis, the findings reveal mixed success, as shown in Figure 1.

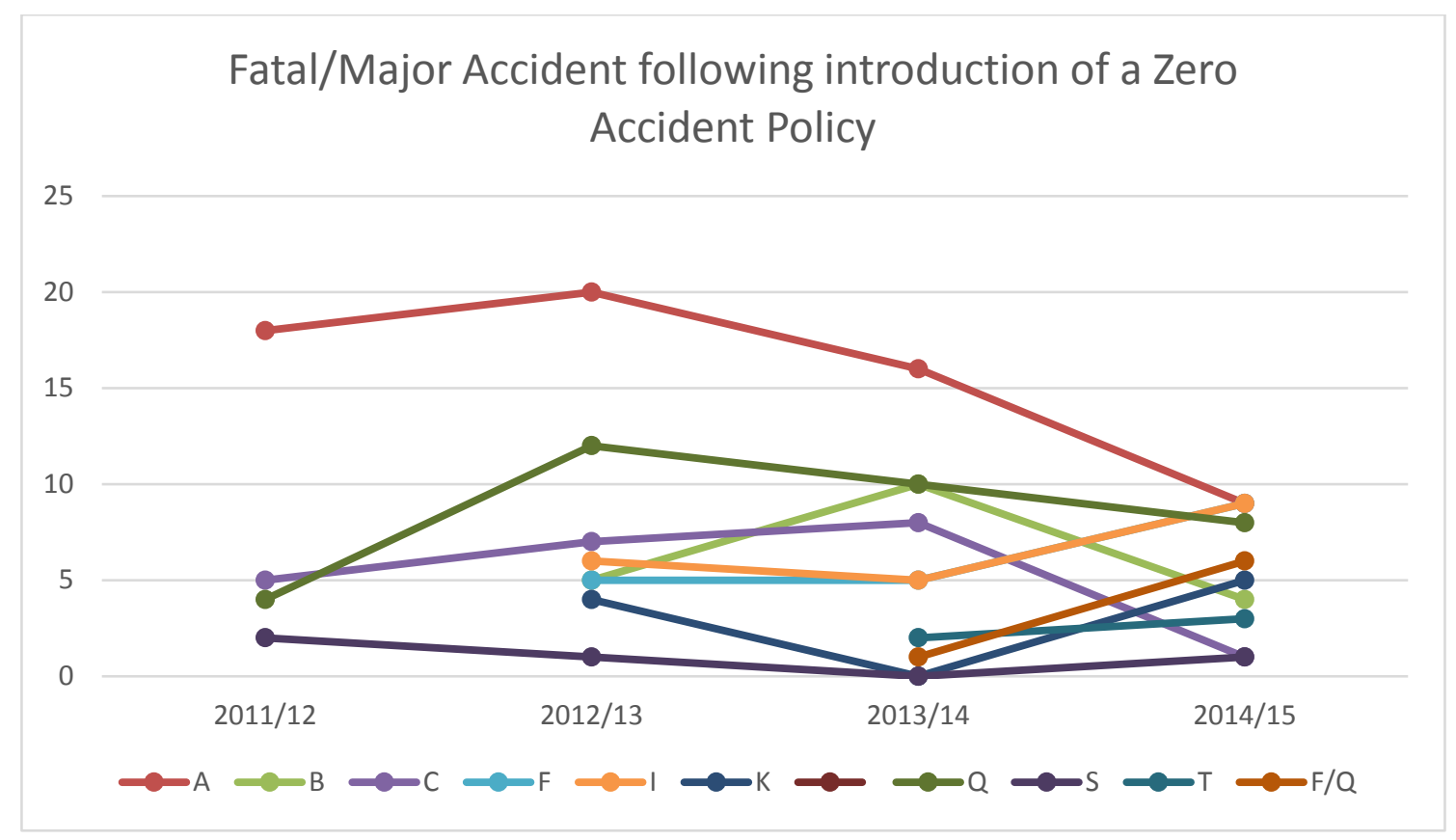

Figure 1

Figure 1 shows that there is no standard response within an organisation's accident profile to the introduction of zero. Indeed, for 7 of the organisations, the 
introduction of zero initially appears to increase accident rates on their sites. Although for 5 organisations this initial peak was then followed by a reduction in accidents, for the other 5 this was not the case and despite early success with zero their accident rates then increased over time. While it must be acknowledged here that this data is limited to only 4 years, it nevertheless suggests that there is at least the potential for an increase in accidents following the introduction of zero safety on sites. There is no guarantee that the implementation of zero safety can ensure continued or even any reduction in accident rates overall, and indeed seems to have limited impact in terms of catalysing any significant step-change improvement in safety performance.

\section{Discussion}

This empirical analysis has shown that the impact of Zero, the expected innovations in the form of changes to programmes and interventions so keenly championed by Zwetsloot et al (2017), are lacking in their impact to practice within the UK construction industry, should they even exist. Indeed the consequences of any Zero safety approach, as evidenced by the corresponding figures for accidents on sites, would struggle to be labelled a success; there are more accidents, not fewer, under the zero safety banner. Fundamentally, this analysis reveals prima facie that the notion that espousing Zero will bring about Zero accidents is flawed.

Yet this is perhaps overemphasising the impact of Zero in practice. In fact, it could also be argued that the introduction of Zero to UK construction safety management does not appear to have made a significant contribution to either any improvement or decline of the industry's safety performance. However such 'nonimpact' can itself be considered worthy of comment. That Zero has not brought about significant change over recent years suggests that the rhetoric of Zero is masking the reality of construction site safety programmes which are neither innovating nor 
developing in their thinking or practice, beyond the application of this new branding. As suggested by critics of Zero, a focus on the numbers and the 'bureaucratization of safety’ has instead created a distraction (Dekker, 2014; Sherratt, 2016b), bringing about Long's (2012) 'paralysis by analysis', and resulting in the failure to bring real change for the people directly affected by poor safety management, a negative consequence of the implementation of Zero in practice.

That Zero has not brought about effective change over the four year period examined here suggests the realisation of some of the more critical challenges made against it. As Long (2012) argued, Zero in practice can easily foster the development of non-learning cultures, resulting in the closing down of debates around safety and a resultant focus on failure. Unfortunately within this dataset, failure is all too apparent, and there has been a lack of coherent, evidenced improvements in safety performance for those operating under the banner of Zero.

It may be that the UK construction industry is simply not yet 'mature' enough for Zero, in either its guise as a target or a vision, to work within its safety management operations. However, when Zero is considered in light of the evidence presented here, it can even be suggested that the dis-benefits outweigh the benefits; Zero is causing more harm than it is able to prevent. Within this industry context, such conclusions add strength to the arguments of Hollnagel (2014) and the need to dismiss Zero, or more specifically the quest for Zero, as a Safety-I myth, a consequence of the now-debunked ‘causality credo’. Instead, Hollnagel proposes Safety-II, a different approach to safety built upon open and honest dialogue and a focus on learning from working safely. The extent to which a more dominant discourse that leans on this hypothesis would realise improvements in safety outcomes remains a moot point, but the open dialogue and recognition of alternative ways of being safe warrant further investigation. Resonance 
can also be found with Hale and Borys' (2013) development of Dekker's models 1 and 2 of safety rules. Model 1, in the way it 'encourages a blame culture and negative view of rules and violations' (ibid 2013, p.218) readily aligns with Zero thinking, whilst model 2 sees safety as a continuous, dynamic social process, open discussions around safety a necessity for effective implementation. Model 1 is also mobilised within the same top-down orthodoxy as Zero, whilst model 2 adopts a bottom-up approach to safety, where Zero struggles to avoid dissonance with those who cannot align it to their lived experiences (Donaldson, 2013; Sherratt, 2014). More specifically, in a construction industry context Gherardi and Nicolini (2002) were able to illuminate the ways in which doing and knowing are intertwined in realising safe working outcomes through communities of practices, supporting the need for flexibility as found in Dekker's Model 2. They stress '...the importance of the conditions and modes of engagement in the activity' (ibid 2002, p.217) as essential for learning safety, a direct challenge to the notion of safe working as a product of the hegemonic discourse of Zero, which is unable to reflect the realities of working safely in practice. And it is here that Zero safety perhaps becomes most relevant in this context, on the sites and amongst the construction workers themselves, and it is here that, to support Dekker et al. (2016), these findings highlight that with Zero safety accidents do still happen, and so organisational efforts should perhaps refocus not at their causes and quixotic quests for their elimination, but more prosaically at their effects.

\section{Conclusions}

Although these findings should be treated with caution, and no claims can be made to causality, this paper makes two important contributions to the ongoing debate around Zero. Firstly, it has introduced the notion of a possible Zero Paradox - the finding that you are actually marginally more likely to have major/specified accident working on a 
large UK construction site operated by a company that has adopted Zero within their safety strategy, than if you are working on a site without it. Secondly, it has also enabled the consideration of theoretical criticisms of Zero to be placed within an empirical context, both in terms of safety outcomes (accident rates) and time. Within the UK construction industry, there is no empirical evidence of greater improvements in safety management and resultant outcomes for companies that have adopted Zero than for those who have not. Zero has arguably stymied innovation and change, or at the very least maintained safety management practices to industry norms with the exception of Zero branding.

These findings also therefore lend weight to the various challenges levelled at Zero; the need for Safety II over Safety I (Hollnagel, 2014), the need to acknowledge Model 2 alongside Model 1 (Hale and Borys, 2013), the need to de-bureaucratise safety (Dekker, 2014; Long, 2012) as well as broader critiques of the Zero mantra (Long, 2012; Sherratt, 2016a). It suggests that it is perhaps time to abandon the flawed discourse of Zero and instead consider and mobilise more contingent, nuanced and authentic approaches to safety in practice.

Whilst theoretical arguments abound about what Zero actually is, a vision or a target, our findings suggest that for the UK construction industry at least, Zero not only hasn't worked, but it has been counterproductive in certain cases. It has arguably not been able to bring about positive change, it has not triggered innovations in practice, and it has not reduced accidents. Further research is therefore recommended to explore why Zero has not found any fit with UK construction sites. Such research should involve those tasked with the implementation of Zero at the site level, where the accidents actually happen, and include site managers, supervisors and workers. Methods able to reveal the nuances and complexities of Zero in practice, for example 
those seeking qualitative data or mobilising ethnographic approaches, would be able to better illuminate its relationships with this uniquely challenging, complex, and everchanging workplace.

\section{References}

Ali, S. (2006). Using visual materials, in Seale, C. (Ed) Researching Society and Culture (pp. 265-278). London: Sage Publications Limited.

Building Magazine (2016). Top 150 contractors and housebuilder - Ranked by Turnover. [Online] Available- http://ubm.io/2dOEq17 [20 June 2016].

Cipolla, D., Sheahan, V.L., Biggs, H. and Dingsdag, D. (2006). Using Safety Culture to overcome market force influence on construction site safety. [Online] Available: http://bit.ly/2fapoTl [02 November 2016].

Dekker, S.W.A. (2014). The bureaucratization of safety. Safety Science. 70. 348-357. http://dx.doi.org/10.1016/j.ssci.2014.07.015

Dekker, S.W.A, Long, R. and Wybo, J-L. (2016). Zero vision and a Western salvation narrative. Safety Science. 88, 219-223. http://dx.doi.org/10.1016/j.ssci.2015.11.016

Donaghy, R. (2009). One Death is too Many - Inquiry into the Underlying Causes of Construction Fatal Accidents. Norwich: Her Majesty’s Stationary Office.

Fellows, R., Langford, D., Newcombe, R. \& Urry, S. (2002). Construction Management in Practice ( $2^{\text {nd }}$ ed). Oxford: Blackwell Science Limited.

Gherardi, S. and Nicolini, D. (2002). Learning the Trade: A Culture of Safety in Practice. Organization. 9(2), 191-223. https://doi.org/10.1177/1350508402009002264

Green, J. (2016). Safety - time for a new direction. EEJ, Laing O’Rourke. [Online] Available: http://bit.ly/2i4PsfY [8 January 2017].

Hale, A. and Borys, D. (2013). Working to rule, or working safely? Part 1: state of the art review. Safety Science. 55, 207-221. http://dx.doi.org/10.1016/j.ssci.2012.05.011

Health and Safety Executive (2009). Phase 2 Report: Underlying causes of construction fatal accidents - Review and sample analysis of recent construction fatal accidents. Norwich: Her Majesty’s Stationary Office. 
Health and Safety Executive (2016). Types of Reportable Incidents. [Online] Available http://bit.ly/2eZ207E [20 June 2016].

Hollnagel, E. (2014). Safety I and Safety II - The Past and Future of Safety Management. Ashgate: Ashgate Publishing Limited.

International Labour Organisation (2008). [Online] Available: http://bit.ly/2fuBTuv [28 October 2016].

Lingard, H. and Rowlinson, S. (Eds) (2005). Occupational Health and Safety in Construction Project Management. London: Spon Press.

Long, R. (2012). The Zero Aspiration, The Maintenance of a Dangerous Idea. Human Dimensions. [Online] Available: http://bit.ly/2fdYs6A [28 Oct 2016].

Long, R. (2012). For the Love of Zero. ACT Australia: Scotoma Press.

Long, R. and Long, J. (2012). Risk Makes Sense. ACT Australia: Scotoma Press.

Manu, P., Ankrah, N., Proverbs, D. and Suresh, S. (2010). An approach for determining the extent of contribution of construction project features to accident causation. Safety Science. 48, 687-692. http://dx.doi.org/10.1016/j.ssci.2010.03.001

Office of National Statistics (2015). Construction Annual Statistics Tables. [Online] Available: http://bit.ly/2fmVsnr [20 July 16].

Pitsis, T. S., Clegg, S. R., Marosszeky, M., \& Rura-Polley, T. (2003). Constructing the Olympic dream: a future perfect strategy of project management. Organization Science. 14(5), 574-590. http://bit.ly/2i2CDI3

Seymour, D. and Fellows, R. (2002) Perspectives of Culture in Construction. CIB Publication No275, CIB, Rotterdam.

Sherratt, F. (2014). Exploring 'Zero Target' safety programmes in the UK construction industry. Construction Management and Economics. 32 (7-8), 737-748. http://dx.doi.org/10.1080/01446193.2014.894248

Sherratt, F. (2016a). Shiny Happy People? UK Construction Industry Health: Priorities, Practice and Public Relations. In: P W Chan and C J Neilson (Eds.) Proceedings of the 32nd Annual ARCOM Conference (Vol 1 487-496). Association of Researchers in Construction Management.

Sherratt, F. (2016b). Unpacking Construction Site Safety. Chichester: John Wiley and Sons.

Smallwood, J. \& Lingard, H. (2009). Occupational health and safety (OH\&S) and corporate social responsibility, In Murray, M. \& Dainty, A.R.J. (Eds). Corporate 
Social Responsibility in the Construction Industry (pp. 261-286). Abingdon: Taylor and Francis.

Young, S. (2014). From zero to hero. A case study of industrial injury reduction: New Zealand Aluminium Smelters Limited. Safety Science. 64, 99-108. http://dx.doi.org/10.1016/j.ssci.2013.11.016

Zwetsloot, G.I.J.M., Aaltonen, M., Wybo, J-L, Saari, J. and Kines, P. (2013). The case for research into the zero accident vision. Safety Science. 58, 41-48. http://dx.doi.org/10.1016/j.ssci.2013.01.026

Zwetsloot, G.I.J.M, Kines, P., Wybo, J-L., Ruotsala, R. and Drupsteen, L. (2017). Zero Accident Vision based strategies in organisations: Innovative perspectives. Safety Science. 91, 260-268. http://dx.doi.org/10.1016/j.ssci.2016.08.016 
Figure 1. Fatal/Major accidents reported by companies following the introduction of zero safety 